\title{
On the Well-Posedness of a Fractional Model of HIV Infection
}

\author{
Mohamed Jleli $\mathbb{D}$ and Bessem Samet $\mathbb{D}$ \\ Department of Mathematics, King Saud University, P.O. Box 2455, Riyadh 11451, Saudi Arabia \\ Correspondence should be addressed to Bessem Samet; bsamet@ksu.edu.sa
}

Received 3 October 2020; Revised 17 October 2020; Accepted 29 October 2020; Published 19 November 2020

Academic Editor: Nawab Hussain

Copyright (c) 2020 Mohamed Jleli and Bessem Samet. This is an open access article distributed under the Creative Commons Attribution License, which permits unrestricted use, distribution, and reproduction in any medium, provided the original work is properly cited.

In this paper, we are concerned with the well-posedness of a fractional model of human immunodeficiency virus infection. Namely, using Grönwall's lemma and Perov's fixed point theorem, we obtain sufficient conditions for which the considered model admits a unique solution.

\section{Introduction}

The human immunodeficiency virus (HIV) is one of the world's leading infectious diseases. A big number of people have died around the globe due to this disease. HIV infects vital cells in the human immune system, such as CD4+ T cells. In this way, the body becomes progressively more susceptible to opportunistic infections, leading to the development of AIDS (acquired immunodeficiency syndrome) (see, e.g., $[1,2])$.

Mathematical models play an important role in understanding the dynamics of HIV infection. The dynamical models for HIV usually consist of systems of ordinary differential equations which range from two-component models (see, e.g., [3, 4]) to three-component (see e.g. [5-7]) and four-component models (see, e.g., [8]). In particular, in [3], the following two-cell model was proposed to describe the HIV infection:

$$
\left\{\begin{array}{l}
u^{\prime}(t)=\kappa-\sigma u(t)-\delta u(t) v(t) \\
v^{\prime}(t)=\delta u(t) v(t)-\tau v(t)
\end{array}\right.
$$

where $u$ is the density of uninfected CD4+ T cells, $v$ is the density of virus-producing cells, $\kappa$ is the rate of production of $\mathrm{CD} 4+\mathrm{T}$ cells, $\sigma$ is their per capita death rate, $\delta$ is the rate of infection of CD4+ T cells, and $\tau$ is the rate of disappearance of infected cells.
Due to the importance of fractional calculus in modeling real-world phenomena (see, e.g., [9-12] and the references therein), a great attention was paid to the study of fractional models of HIV infection of CD4+ T cells (see, e.g., [13-17] and the references therein).

In this paper, a fractional model of (1) is investigated. Namely, we are concerned with the system of fractional differential equations

$$
\begin{cases}\left({ }^{C} D_{0}^{\alpha, \psi} u\right)(t)=I_{0}^{\mu, \psi}(\kappa-\sigma u-\delta u v)(t), & 0 \leq t \leq T, \\ \left({ }^{C} D_{0}^{\beta, \psi} v\right)(t)=I_{0}^{v, \psi}(\delta u v-\tau v)(t), & 0 \leq t \leq T,\end{cases}
$$

subjet to the initial conditions

$$
(u(0), v(0))=\left(u_{0}, v_{0}\right)
$$

where $T>0, u_{0}, v_{0} \geq 0,0<\alpha, \beta<1, \mu \geq 1-\alpha, v \geq 1-\beta, \psi \in$ $C^{1}([0, T]), \psi^{\prime}>0,{ }^{C} D_{0}^{\ell, \psi}, \ell \in\{\alpha, \beta\}$ is the $\psi$-Caputo fractional derivative of order $\ell, I_{0}^{\varkappa, \psi}, \varkappa \in\{\mu, \nu\}$ is the $\psi$-Riemann-Liouville fractional integral of order $\mathrm{u}$, and $\kappa, \sigma, \delta, \tau>0$. Using Grönwall's lemma and Perov's fixed point theorem, we derive sufficient conditions for which system (2) subject to the initial conditions (3) admits a unique solution. Moreover, we provide a numerical algorithm that converges uniformly to the unique solution. Notice that 
when $\psi(t)=t,(\alpha, \mu) \rightarrow\left(1^{-}, 0^{+}\right)$and $(\beta, v) \rightarrow\left(1^{-}, 0^{+}\right)$, (2) reduces to (1).

The rest of the paper is organized as follows. In Section 2, we recall some notions on fractional calculus and Perov's fixed point theorem. In Section 3, we state and prove our main results. In Section 4, some special cases are discussed.

\section{Some Preliminaries}

Let $(a, b) \in \mathbb{R}^{2}$ be such that $a<b$.

Given $f \in C([a, b])$ and $\theta>0$, the (left-sided) RiemannLiouville fractional integral of order $\theta$ of $f$ is defined by (see [10])

$$
\left(I_{a}^{\theta} f\right)(t)=\left\{\begin{array}{lll}
\frac{1}{\Gamma(\theta)} \int_{a}^{t}(t-s)^{\theta-1} f(s) d s & \text { if } & a<t \leq b \\
0 & \text { if } \quad t=a
\end{array}\right.
$$

where $\Gamma$ is the Gamma function. It can be easily seen that

$$
f \in C([a, b]) \Longrightarrow I_{a}^{\theta} f \in C([a, b]) .
$$

Lemma 1 (see [10], Property 2.6). Let $f \in C([a, b])$ and $\theta, \eta$ $>0$. Then,

$$
\left(I_{a}^{\theta} I_{a}^{\eta} f\right)(t)=\left(I_{a}^{\theta+\eta} f\right)(t), \quad a \leq t \leq b .
$$

Given $f \in C^{1}([a, b])$ and $0<\theta<1$, the (left-sided) Caputo fractional derivative of order $\theta$ of $f$ is defined by (see [10])

$$
\left({ }^{C} D_{a}^{\theta} f\right)(t)=\left(I_{a}^{1-\theta} f^{\prime}\right)(t), \quad a \leq t \leq b .
$$

Lemma 2 (see [10], Lemma 2.22). Let $f \in C^{1}([a, b])$ and $0<$ $\theta<1$. Then,

$$
\left(I_{a}^{\theta^{C}} D_{a}^{\theta} f\right)(t)=f(t)-f(a), \quad a \leq t \leq b .
$$

Lemma 3 (see [10], Lemma 2.21). Let $f \in C([a, b])$ and $0<$ $\theta<1$. Then,

$$
\left({ }^{C} D_{a}^{\theta} I_{a}^{\theta} f\right)(t)=f(t), a \leq t \leq b .
$$

Let $T>0$ be fixed. We introduce the set of functions

$$
\Psi=\left\{\psi \in C^{l}([0, T]): \psi^{\prime}(t)>\text { for all } 0 \leq t \leq T\right\} .
$$

Given $f \in C([0, T]), \theta>0$, and $\psi \in \Psi$, the (left-sided) $\psi$ -Riemann-Liouville fractional integral of order $\theta$ of $f$ is defined by (see $[10,18])$

$$
\left(I_{0}^{\theta, \psi} f\right)(t)= \begin{cases}\frac{1}{\Gamma(\theta)} \int_{0}^{t}(\psi(t)-\psi(s))^{\theta-1} \psi^{\prime}(s) f(s) d s & \text { if } \quad 0<t \leq T \\ 0 & \text { if } \quad t=0 .\end{cases}
$$

It can be easily seen that

$$
f \in C([0, T]) \Rightarrow I_{0}^{\theta, \psi} f \in C([0, T]) .
$$

Remark 4. In the special case $\psi(t)=t$, one observes that $I_{0}^{\theta, \psi}$ $=I_{0}^{\theta}$.

Remark 5. Using the change of variable $z=\psi(s)$, one deduces from ((11)) that

$$
\left(I_{0}^{\theta, \psi} f\right)(t)=\left(I_{\psi(0)}^{\theta} f \circ \psi^{-1}\right)(\psi(t)), 0 \leq t \leq T .
$$

Using Lemma 1 and Remark 5, one deduces the following result.

Lemma 6. Let $f \in C([0, T]), \theta, \eta>0$ and $\psi \in \Psi$. Then

$$
\left(I_{0}^{\theta, \psi} I_{0}^{\eta, \psi} f\right)(t)=\left(I_{0}^{\theta+\eta, \psi} f\right)(t), 0 \leq t \leq T .
$$

Given $f \in C^{1}([0, T]), 0<\theta<1$, and $\psi \in \Psi$, the (left-sided) $\psi$-Caputo fractional derivative of order $\theta$ of $f$ is defined by (see [18])

$$
\left({ }^{C} D_{0}^{\theta, \psi} f\right)(t)=\left(I_{0}^{1-\theta, \psi} \frac{f^{\prime}}{\psi^{\prime}}\right)(t), 0 \leq t \leq T .
$$

Remark 7. In the special case $\psi(t)=t$, one observes that ${ }^{C}$ $D_{0}^{\theta, \psi}={ }^{C} D_{0}^{\theta}$.

Remark 8. Using the change of variable $z=\psi(s)$, one deduces from ((15)) that

$$
\left({ }^{C} D_{0}^{\theta, \psi} f\right)(t)=\left({ }^{C} D_{\psi(0)}^{\theta} f \circ \psi^{-1}\right)(\psi(t)), 0 \leq t \leq T .
$$

Using Lemma 2, Remark 5, and Remark 8, one deduces the following result.

Lemma 9. Let $f \in C^{1}([0, T]), 0<\theta<1$ and $\psi \in \Psi$. Then

$$
\left(I_{0}^{\theta, \psi}{ }^{C} D_{0}^{\theta, \psi} f\right)(t)=f(t)-f(0), 0 \leq t \leq T .
$$

Similarly, using Lemma 3, Remark 5, and Remark 8, one deduces the following result.

Lemma 10. Let $f \in C([0, T]), 0<\theta<1$ and $\psi \in \Psi$. Then,

$$
\left({ }^{C} D_{0}^{\theta, \psi} I_{0}^{\theta, \psi} f\right)(t)=f(t), 0 \leq t \leq T .
$$


Now, we recall some concepts on fixed point theory that will be used later. Let $n$ be a positive natural number and define the partial order ${ }_{n}$ in $\mathbb{R}^{n}$ by

$y=\left(\begin{array}{c}y_{1} \\ y_{2} \\ \vdots \\ y_{n}\end{array}\right) \preceq_{n} z=\left(\begin{array}{c}z_{1} \\ z_{2} \\ \vdots \\ z_{n}\end{array}\right) \Leftrightarrow y_{i} \leq z_{i}, i=1,2, \cdots, n$,

for all $y, z \in \mathbb{R}^{n}$. We denote by $0_{\mathbb{R}^{n}}$ the zero vector in $\mathbb{R}^{n}$, i.e.,

$$
0_{\mathbb{R}^{n}}=\left(\begin{array}{c}
0 \\
0 \\
\vdots \\
0
\end{array}\right)
$$

Let $\mathscr{X}$ be a nonempty set and $d: \mathscr{X} \times \mathscr{X} \rightarrow \mathbb{R}^{n}$ be a given mapping. We say that $d$ is a vector-valued metric on $\mathscr{X}$ (see, e.g., [19]), if for all $x, y, z \in \mathscr{X}$,

$$
\begin{gathered}
0_{\mathbb{R}^{n}} \varliminf_{n} d(x, y), \\
d(x, y)=0_{\mathbb{R}^{n}} \Leftrightarrow x=y, \\
d(x, y)=d(y, x), \\
d(x, z) \varliminf_{n} d(x, y)+d(y, z) .
\end{gathered}
$$

In this case, the pair $(\mathscr{X}, d)$ is called a generalized metric space. In such spaces, the notions of convergent sequence, Cauchy sequence, and completeness are similar to those for usual metric spaces.

Let us denote by $M_{n}\left(\mathbb{R}_{+}\right)$the set of square matrices of size $n$ with nonnegative coefficients. Given $M \in M_{n}\left(\mathbb{R}_{+}\right)$, we denote by $\rho(M)$ its spectral radius.

Lemma 11 (Perov's fixed point theorem). Let $(\mathscr{X}, d)$ be a complete generalized metric space and $F: \mathscr{X} \rightarrow \mathscr{X}$ be a given mapping. Suppose that there exists $\mathscr{M} \in M_{n}\left(\mathbb{R}_{+}\right)$with $\rho(M)<1$ such that

$$
d(F(x), F(y)) \underline{n}_{n} \mathscr{M} d(x, y),
$$

for all $x, y \in \mathscr{X}$. Then,

(i) the mapping $F$ admits a unique fixed point in $\mathscr{X}$, say $x^{*}$

(ii) for all $x_{0} \in \mathscr{X}$, the sequence $\left\{x_{m}\right\} \subset \mathscr{X}$ defined by $x_{m+1}=F\left(x_{m}\right)$ converges to $x^{*}$

We end this section by recalling the Grönwall's lemma.
Lemma 12. (see [20]) Let $K \geq 0$ and $f, g$ be nonnegative functions on $[0, T]$ such that $f \in L^{\infty}(0, T)$ and $g \in L^{1}(0, T)$. If

$$
f(t) \leq K+\int_{0}^{t} g(s) f(s) d s, 0 \leq t \leq T,
$$

then,

$$
f(t) \leq K \exp \left(\int_{0}^{t} g(s) d s\right), 0 \leq t \leq T .
$$

\section{Main Results}

Problem (2) and (3) is investigated under the following assumptions:

(i) $T>0$ and $\psi \in \Psi$, where $\Psi$ is the functional space defined by (10).

(ii) $u_{0}, v_{0} \geq 0$ and $\kappa, \sigma, \delta, \tau>0$

(iii) $0<\alpha<1$ and $\mu \geq 1-\alpha$

(iv) $0<\beta<1$ and $v \geq 1-\beta$

3.1. Integral Formulation of Problem (2) and (3). Let

$$
\begin{gathered}
V=\{(u, v) \in C([0, T]) \times C([0, T]): u, v \geq 0\} \\
W=\left\{(u, v) \in C^{1}([0, T]) \times C^{1}([0, T]): u, v \geq 0\right\} .
\end{gathered}
$$

Suppose that $(u, v) \in W$ is a solution to problem (2) and (3). Using the first equation in (2), one obtains

$$
\left(I_{0}^{\alpha, \psi C} D_{0}^{\alpha, \psi} u\right)(t)=\left(I_{0}^{\alpha, \psi} I_{0}^{\mu, \psi}(\kappa-\sigma u-\delta u v)\right)(t), 0 \leq t \leq T .
$$

Hence, by Lemma 6 and Lemma 9, it holds that

$$
u(t)-u(0)=I_{0}^{\alpha+\mu, \psi}(\kappa-\sigma u-\delta u v)(t), 0 \leq t \leq T .
$$

Using the initial conditions (3), one obtains

$$
\begin{gathered}
u(t)=u_{0}+\frac{1}{\Gamma(\alpha+\mu)} \int_{0}^{t}(\psi(t)-\psi(s))^{\alpha+\mu-1} \psi^{\prime}(s) \\
\cdot(\kappa-\sigma u(s)-\delta u(s) v(s)) d s, 0 \leq t \leq T .
\end{gathered}
$$

Similarly, using the second equation in (2), one obtains

$$
\left(I_{0}^{\beta, \psi^{C}} D_{0}^{\beta, \psi} v\right)(t)=\left(I_{0}^{\beta, \psi} I_{0}^{v, \psi}(\delta u v-\tau v)\right)(t), 0 \leq t \leq T,
$$

which yields

$$
v(t)-v(0)=I_{0}^{\beta+v, \psi}(\delta u v-\tau v)(t), 0 \leq t \leq T .
$$


By the initial conditions (3), it holds that

$$
\begin{aligned}
& v(t)= v_{0}+\frac{1}{\Gamma(\beta+v)} \int_{0}^{t}(\psi(t)-\psi(s))^{\beta+v-1} \psi^{\prime}(s) \\
& \cdot(\delta u(s) v(s)-\tau v(s)) d s, 0 \leq t \leq T .
\end{aligned}
$$

Therefore, one deduces that, if $(u, v) \in W$ is a solution to problem (2) and (3), then $(u, v) \in V$ is a solution to the system of integral equations

$$
\left\{\begin{array}{l}
u(t)=u_{0}+\frac{1}{\Gamma(\alpha+\mu)} \int_{0}^{t}(\psi(t)-\psi(s))^{\alpha+\mu-1} \psi^{\prime}(s)(\kappa-\sigma u(s)-\delta u(s) v(s)) d s \\
v(t)=v_{0}+\frac{1}{\Gamma(\beta+v)} \int_{0}^{t}(\psi(t)-\psi(s))^{\beta+v-1} \psi^{\prime}(s)(\delta u(s) v(s)-\tau v(s)) d s
\end{array}\right.
$$

for all $0 \leq t \leq T$.

Conversely, suppose that $(u, v) \in V$ is a solution to (32). By assumptions (iii) and (iv), one deduces that $(u, v) \in W$. Moreover, by (32), one has $u(0)=u_{0}$ and $v(0)=v_{0}$. On the other hand, using the first equation in (32), Lemma 6, and Lemma 10, one obtains

$$
\begin{aligned}
\left({ }^{C} D_{0}^{\alpha, \psi} u\right)(t) & =\left({ }^{C} D_{0}^{\alpha, \psi} I_{0}^{\alpha+\mu, \psi}(\kappa-\sigma u-\delta u v)\right)(t) \\
& =\left({ }^{C} D_{0}^{\alpha, \psi} I_{0}^{\alpha, \psi} I_{0}^{\mu, \psi}(\kappa-\sigma u-\delta u v)\right)(t) \\
& =I_{0}^{\mu, \psi}(\kappa-\sigma u-\delta u v)(t) .
\end{aligned}
$$

Similarly, using the second equation in (32), one obtains

$$
\begin{aligned}
\left({ }^{C} D_{0}^{\beta, \psi} v\right)(t) & =\left({ }^{C} D_{0}^{\beta, \psi} I_{0}^{\beta+v, \psi}(\delta u v-\tau v)\right)(t) \\
& =\left({ }^{C} D_{0}^{\beta, \psi} I_{0}^{\beta, \psi} I_{0}^{v, \psi}(\delta u v-\tau v)\right)(t) \\
& =I_{0}^{v, \psi}(\delta u v-\tau v)(t) .
\end{aligned}
$$

Therefore, one deduces that, if $(u, v) \in V$ is a solution to the system of integral equation (32), then $(u, v) \in W$ is a solution to problem (2) and (3).

From the above study, the following result holds.

Lemma 13. The following statements are equivalent:

(I) $(u, v) \in W$ is a solution to problem (2) and (3).

(II) $(u, v) \in V$ is a solution to the system of integral equations (32).

By the above lemma, the study of problem (2) and (3) in $W$ reduces to the study of the system of integral equation (32) in $V$.

3.2. Uniqueness. In this part, using Grönwall's lemma, we shall prove that the system of integral equations (32) admits at most one solution $(u, v) \in V$.
Proposition 14. Suppose that the assumptions (i)-(iv) are satisfied. Then the system of integral equation ((32)) admits at most one solution $(u, v) \in V$.

Proof. Suppose that $\left(u_{1}, v_{1}\right),\left(u_{2}, v_{2}\right) \in V$ are two solutions to (32). Then, for all $0 \leq t \leq T$, one has

$$
\begin{aligned}
u_{2}(t)-u_{1}(t)= & u_{0}+\frac{1}{\Gamma(\alpha+\mu)} \int_{0}^{t}(\psi(t)-\psi(s))^{\alpha+\mu-1} \psi^{\prime}(s) \\
& \cdot\left(\kappa-\sigma u_{2}(s)-\delta u_{2}(s) v_{2}(s)\right) d s-u_{0} \\
& -\frac{1}{\Gamma(\alpha+\mu)} \int_{0}^{t}(\psi(t)-\psi(s))^{\alpha+\mu-1} \psi^{\prime}(s) \\
& \cdot\left(\kappa-\sigma u_{1}(s)-\delta u_{1}(s) v_{1}(s)\right) d s \\
= & \frac{\sigma}{\Gamma(\alpha+\mu)} \int_{0}^{t}(\psi(t)-\psi(s))^{\alpha+\mu-1} \psi^{\prime}(s) \\
& \cdot\left(u_{1}(s)-u_{2}(s)\right) d s \\
& +\frac{\delta}{\Gamma(\alpha+\mu)} \int_{0}^{t}(\psi(t)-\psi(s))^{\alpha+\mu-1} \psi^{\prime}(s) \\
& \cdot\left(u_{1}(s) v_{1}(s)-u_{2}(s) v_{2}(s)\right) d s,
\end{aligned}
$$

which yields

$$
\begin{aligned}
\left|u_{2}(t)-u_{1}(t)\right| \leq & \sigma C_{1} \int_{0}^{t} \psi^{\prime}(s)\left|u_{1}(s)-u_{2}(s)\right| d s \\
& +\delta C_{1} \int_{0}^{t} \psi^{\prime}(s)\left|u_{1}(s) v_{1}(s)-u_{2}(s) v_{2}(s)\right| d s \\
= & \sigma C_{1} \int_{0}^{t} \psi^{\prime}(s)\left|u_{1}(s)-u_{2}(s)\right| d s \\
& +\delta C_{1} \int_{0}^{t} \psi^{\prime}(s) \mid u_{1}(s)\left(v_{1}(s)-v_{2}(s)\right) \\
& +v_{2}(s)\left(u_{1}(s)-u_{1}(s)\right) \mid d s \\
\leq & \sigma C_{1} \int_{0}^{t} \psi^{\prime}(s)\left|u_{1}(s)-u_{2}(s)\right| d s \\
& +\delta C_{1} C_{2} \int_{0}^{t} \psi^{\prime}(s)\left|v_{1}(s)-v_{2}(s)\right| d s \\
& +\delta C_{1} C_{3} \int_{0}^{t} \psi^{\prime}(s)\left|u_{1}(s)-u_{2}(s)\right| d s \\
= & C_{1}\left(\sigma+\delta C_{3}\right) \int_{0}^{t} \psi^{\prime}(s)\left|u_{1}(s)-u_{2}(s)\right| d s \\
& +\delta C_{1} C_{2} \int_{0}^{t} \psi^{\prime}(s)\left|v_{1}(s)-v_{2}(s)\right| d s
\end{aligned}
$$

where

$$
C_{1}=\frac{(\psi(T)-\psi(0))^{\alpha+\mu-1}}{\Gamma(\alpha+\mu)}, C_{2}=\max _{0 \leq x \leq T} u_{1}(x), C_{3}=\max _{0 \leq x \leq T} v_{2}(x)
$$


Hence, one deduces that

$$
\begin{aligned}
\left|u_{2}(t)-u_{1}(t)\right| \leq & C_{4} \int_{0}^{t} \psi^{\prime}(s)\left(\left|u_{1}(s)-u_{2}(s)\right|\right. \\
& \left.+\left|v_{1}(s)-v_{2}(s)\right|\right) d s
\end{aligned}
$$

where

$$
C_{4}=C_{1} \max \left\{\sigma+\delta C_{3}, \delta C_{2}\right\}
$$

Similarly, for all $0 \leq t \leq T$, one has

$$
\begin{aligned}
v_{2}(t)-v_{1}(t)= & v_{0}+\frac{1}{\Gamma(\beta+v)} \int_{0}^{t}(\psi(t)-\psi(s))^{\beta+v-1} \psi^{\prime}(s) \\
& \cdot\left(\delta u_{2}(s) v_{2}(s)-\tau v_{2}(s)\right) d s-v_{0} \\
& -\frac{1}{\Gamma(\beta+v)} \int_{0}^{t}(\psi(t)-\psi(s))^{\beta+v-1} \psi^{\prime}(s) \\
& \cdot\left(\delta u_{1}(s) v_{1}(s)-\tau v_{1}(s)\right) d s \\
= & \frac{\delta}{\Gamma(\beta+v)} \int_{0}^{t}(\psi(t)-\psi(s))^{\beta+v-1} \psi^{\prime}(s) \\
& \cdot\left(u_{2}(s) v_{2}(s)-u_{1}(s) v_{1}(s)\right) d s \\
& +\frac{\tau}{\Gamma(\beta+v)} \int_{0}^{t}(\psi(t)-\psi(s))^{\beta+v-1} \psi^{\prime}(s) \\
& \cdot\left(v_{1}(s)-v_{2}(s)\right) d s,
\end{aligned}
$$

which yields

$$
\begin{aligned}
\left|v_{2}(t)-v_{1}(t)\right|+ & \tau C_{5} \int_{0}^{t} \psi^{\prime}(s)\left|v_{1}(s)-v_{2}(s)\right| d s \\
& \leq \delta C_{5} C_{6} \int_{0}^{t} \psi^{\prime}(s)\left|v_{1}(s)-v_{2}(s)\right| d s \\
+ & \delta C_{5} C_{7} \int_{0}^{t} \psi^{\prime}(s)\left|u_{2}(s)-u_{1}(s)\right| d s \\
+ & \tau C_{5} \int_{0}^{t} \psi^{\prime}(s)\left|v_{1}(s)-v_{2}(s)\right| d s \\
& =C_{5}\left(\delta C_{6}+\tau\right) \int_{0}^{t} \psi^{\prime}(s)\left|v_{1}(s)-v_{2}(s)\right| d s \\
& +\delta C_{5} C_{7} \int_{0}^{t} \psi^{\prime}(s)\left|u_{2}(s)-u_{1}(s)\right| d s,
\end{aligned}
$$

where

$$
C_{5}=\frac{(\psi(T)-\psi(0))^{\beta+\nu-1}}{\Gamma(\beta+v)}, C_{6}=\max _{0 \leq x \leq T} u_{2}(x), C_{7}=\max _{0 \leq x \leq T} v_{1}(x) .
$$

Therefore, one obtains

$$
\left|v_{2}(t)-v_{1}(t)\right| \leq C_{8} \int_{0}^{t} \psi^{\prime}(s)\left(\left|u_{1}(s)-u_{2}(s)\right|+\left|v_{1}(s)-v_{2}(s)\right|\right) d s,
$$

where

$$
C_{8}=C_{5} \max \left\{\tau+\delta C_{6}, \delta C_{7}\right\} .
$$

Next, combining (38) with (43), it holds that

$$
\begin{aligned}
&\left|u_{2}(t)-u_{1}(t)\right|+\mid\left|v_{2}(t)-v_{1}(t)\right| \leq\left(C_{4}+C_{8}\right) \int_{0}^{t} \psi^{\prime}(s) \\
& \cdot\left(\left|u_{2}(s)-u_{1}(s)\right|+\left|v_{2}(s)-v_{2}(s)\right|\right) d s .
\end{aligned}
$$

Finally, using Grönwall 's lemma (see Lemma 12), it holds that $\left(u_{1}, v_{1}\right)=\left(u_{2}, v_{2}\right)$.

3.3. Well-Posedness. We first fix some notations. Let

$$
\begin{aligned}
& a_{T}=\frac{(\psi(T)-\psi(0))^{\alpha+\mu}}{\Gamma(\alpha+\mu+1)}, \\
& b_{T}=\frac{(\psi(T)-\psi(0))^{\beta+\nu}}{\Gamma(\beta+v+1)} .
\end{aligned}
$$

We introduce the function $f:[0, \infty) \rightarrow[0, \infty)$ defined by

$$
\begin{aligned}
f(r)= & a_{T} \sigma+b_{T} \tau+\left(a_{T}+b_{T}\right) \delta r+\left[\left(\left(a_{T} \sigma-b_{T} \tau\right)\right.\right. \\
& \left.\left.+\left(a_{T}-b_{T}\right) \delta r\right)^{2}+4 a_{T} b_{T} \delta^{2} r^{2}\right]^{1 / 2}, r \geq 0 .
\end{aligned}
$$

We denote by $\|\cdot\|_{\infty}$ the norm in $C([0, T])$ defined by

$$
\|\xi\|_{\infty}=\max _{0 \leq x \leq T}|\xi(x)|, \xi \in C([0, T]) .
$$

In addition to the assumptions (i)-(iv), suppose that

(v) $a_{T} \leq \tau / \delta \kappa$ and $b_{T} \leq 1 /\left(\sqrt{ }\left(\sigma^{2}+4 \delta \kappa\right)-\sigma\right)$

$$
4 \tau \leq \sqrt{\sigma^{2}+4 \delta \kappa}-\sigma
$$

(vi) There exists

$$
\frac{2 \tau}{\delta} \leq r \leq \frac{\sqrt{\sigma^{2}+4 \delta \kappa}-\sigma}{2 \delta}
$$

such that

$$
\frac{\tau}{\delta} \leq u_{0} \leq \frac{r}{2}, 0 \leq v_{0} \leq \frac{r}{2}, \quad f(r)<2 .
$$


Theorem 15. Suppose that the assumptions (i)-(vii) are satisfied. Then, the system of integral equation ((32)) admits one and only one solution $\left(u^{*}, v^{*}\right) \in V$. Moreover, for all $f_{0}, g_{0}$ $\in V$ satisfying $u_{0} \leq f_{0},\left\|f_{0}\right\|_{\infty} \leq r$ and $\left\|g_{0}\right\|_{\infty} \leq r$, the sequence $\left\{\left(f_{n}, g_{n}\right)\right\} \subset V$ defined by

$\left\{\begin{array}{l}f_{n+1}(t)=u_{0}+\frac{1}{\Gamma(\alpha+\mu)} \int_{0}^{t}(\psi(t)-\psi(s))^{\alpha+\mu-1} \psi^{\prime}(s)\left(\kappa-\sigma f_{n}(s)-\delta f_{n}(s) g_{n}(s)\right) d s, \\ g_{n+1}(t)=v_{0}+\frac{1}{\Gamma(\beta+v)} \int_{0}^{t}(\psi(t)-\psi(s))^{\beta+v-1} \psi^{\prime}(s)\left(\delta f_{n}(s) g_{n}(s)-\tau g_{n}(s)\right) d s,\end{array}\right.$

for all $0 \leq t \leq T$, converges uniformly to $\left(u^{*}, v^{*}\right)$.

Proof. We first introduce the functional space

$$
V_{r}=\left\{(u, v) \in V: u_{0} \leq u,\|u\|_{\infty} \leq r,\|v\|_{\infty} \leq r\right\}
$$

Let $F: V_{r} \rightarrow C([0, T]) \times C([0, T])$ be the mapping defined by

$$
F(u, v)(t)=\left(F_{1}(u, v)(t), F_{2}(u, v)(t)\right), \quad 0 \leq t \leq T,
$$

where

$$
\begin{aligned}
F_{1}(u, v)(t)= & u_{0}+\frac{1}{\Gamma(\alpha+\mu)} \int_{0}^{t}(\psi(t)-\psi(s))^{\alpha+\mu-1} \psi^{\prime}(s) \\
& \cdot(\kappa-\sigma u(s)-\delta u(s) v(s)) d s, \\
F_{2}(u, v)(t)= & v_{0}+\frac{1}{\Gamma(\beta+v)} \int_{0}^{t}(\psi(t)-\psi(s))^{\beta+v-1} \psi^{\prime}(s) \\
& \cdot(\delta u(s) v(s)-\tau v(s)) d s .
\end{aligned}
$$

We shall prove that

$$
F\left(V_{r}\right) \subset V_{r}
$$

Let $(u, v) \in V_{r}$. Then, for all $s \in[0, T]$, one has

$$
\kappa-\sigma u(s)-\delta u(s) v(s) \geq \kappa-\sigma r-\delta r^{2}:=P(r) .
$$

On the other hand, an elementary calculation shows that the polynomial function $P(r)$ admits two roots

$$
r_{1}=\frac{-\sigma-\sqrt{\sigma^{2}+4 \delta \kappa}}{2 \delta}<0<r_{2}=\frac{\sqrt{\sigma^{2}+4 \delta \kappa}-\sigma}{2 \delta}
$$

Since $-\delta<0$ and (by (vii))

$$
0<r \leq \frac{\sqrt{\sigma^{2}+4 \delta \kappa}-\sigma}{2 \delta}=r_{2} \text {, }
$$

one obtains

$$
P(r) \geq 0,
$$

which yields

$$
\kappa-\sigma u(s)-\delta u(s) v(s) \geq 0,0 \leq s \leq T
$$
that

Therefore, since $\psi \in \Psi$ and $u_{0} \geq 0$ (see (vii)), one deduces

$$
F_{1}(u, v) \geq u_{0} \geq 0
$$

Moreover, for all $s \in[0, T]$, one has

$$
\delta u(s) v(s)-\tau v(s)=v(s)(\delta u(s)-\tau) \geq v(s)\left(\delta u_{0}-\tau\right) .
$$

Since by (vii), $u_{0} \geq \tau / \delta$, one obtains

$$
\delta u(s) v(s)-\tau v(s) \geq 0, \quad 0 \leq s \leq T .
$$

Using the above inequality and the fact that $v_{0} \geq 0$ (by (vii)), one deduces that

$$
F_{2}(u, v) \geq 0
$$

On the other hand, by (62), for all $0 \leq t \leq T$, one has

$$
\begin{aligned}
\left|F_{1}(u, v)(t)\right| & =F_{1}(u, v)(t) \\
& \leq u_{0}+\frac{\kappa}{\Gamma(\alpha+\mu)} \int_{0}^{t}(\psi(t)-\psi(s))^{\alpha+\mu-1} \psi^{\prime}(s) d s \\
& =u_{0}+\kappa \frac{(\psi(t)-\psi(0))^{\alpha+\mu}}{\Gamma(\alpha+\mu+1)} \leq u_{0}+\kappa a_{T} .
\end{aligned}
$$
has

Since $a_{T} \leq \tau / \delta \kappa($ by $(\mathrm{v}))$ and $\tau / \delta \leq u_{0} \leq r / 2$ (by (vii)), one

$$
u_{0}+\kappa a_{T} \leq \frac{r}{2}+\frac{\tau}{\delta} \leq \frac{r}{2}+\frac{r}{2}=r
$$

Hence, it follows from (66) and (67) that

$$
\left\|F_{1}(u, v)\right\|_{\infty} \leq r
$$

Similarly, by (65), for all $0 \leq t \leq T$, one has

$$
\begin{aligned}
\left|F_{2}(u, v)(t)\right| & =F_{2}(u, v)(t) \\
& \leq v_{0}+\frac{\delta r^{2}}{\Gamma(\beta+v)} \int_{0}^{t}(\psi(t)-\psi(s))^{\beta+v-1} \psi^{\prime}(s) d s \\
& =v_{0}+\delta r^{2} \frac{(\psi(t)-\psi(0))^{\beta+v}}{\Gamma(\beta+v+1)} \leq v_{0}+\delta r^{2} b_{T} .
\end{aligned}
$$

Since $v_{0} \leq r / 2$ (by (vii)), $b_{T} \leq 1 /\left(\sqrt{ }\left(\sigma^{2}+4 \delta \kappa\right)-\sigma\right.$ ) (by (v)) and $r \leq\left(\sqrt{ }\left(\sigma^{2}+4 \delta \kappa\right)-\sigma\right) / 2 \delta$ (by (vii)), one has

$$
v_{0}+\delta r^{2} b_{T} \leq \frac{r}{2}+\delta r^{2} \frac{1}{2 \delta r}=\frac{r}{2}+\frac{r}{2}=r .
$$


Therefore, it follows from (69) and (70) that

$$
\left\|F_{2}(u, v)\right\|_{\infty} \leq r .
$$

Hence, by (62), (65), (68), and (71), one deduces that $F$ $(u, v) \in V_{r}$. This proves (56).

Consider now the self-mapping,

$$
F: V_{r} \rightarrow V_{r}
$$

By the definition of $F$, one observes that, if $(u, v) \in V_{r}$ is a fixed point of $F$; then, $(u, v) \in V$ is a solution to the system of integral equation (32). In order to prove that $F$ admits a fixed point in $V_{r}$, we shall use Perov's fixed point theorem (see Lemma 11). Namely, we define the vector-valued metric $d$ $: V_{r} \times V_{r} \rightarrow \mathbb{R}^{2}$ by

$d\left(\left(u_{1}, v_{1}\right),\left(u_{2}, v_{2}\right)\right)=\left(\begin{array}{c}\left\|u_{1}-u_{2}\right\|_{\infty} \\ \left\|v_{1}-v_{2}\right\|_{\infty}\end{array}\right),\left(u_{1}, v_{1}\right),\left(u_{2}, v_{2}\right) \in V_{r}$.

Notice that $\left(V_{r}, d\right)$ is a complete generalized metric space. On the other hand, for all $\left(u_{1}, v_{1}\right),\left(u_{2}, v_{2}\right) \in V_{r}$ and $0 \leq t \leq T$, one has

$$
\begin{aligned}
\left|F_{1}\left(u_{2}, v_{2}\right)(t)-F_{1}\left(u_{1}, v_{1}\right)(t)\right| & \\
\leq & \frac{\sigma}{\Gamma(\alpha+\mu)} \int_{0}^{t}(\psi(t)-\psi(s))^{\alpha+\mu-1} \psi^{\prime}(s)\left|u_{1}(s)-u_{2}(s)\right| d s \\
& +\frac{\delta}{\Gamma(\alpha+\mu)} \int_{0}^{t}(\psi(t)-\psi(s))^{\alpha+\mu-1} \psi^{\prime}(s) \mid u_{1}(s) v_{1}(s) \\
& -u_{2}(s) v_{2}(s) \mid d s \leq \sigma a_{T}\left\|u_{1}-u_{2}\right\|_{\infty} \\
& +\frac{\delta}{\Gamma(\alpha+\mu)} \int_{0}^{t}(\psi(t)-\psi(s))^{\alpha+\mu-1} \psi^{\prime}(s) \\
& \times\left(\left|u_{1}(s)\left\|v_{1}(s)-v_{2}(s)|+| v_{2}(s)\right\| u_{2}(s)-u_{1}(s)\right|\right) d s \\
\leq & \sigma a_{T}\left\|u_{1}-u_{2}\right\|_{\infty}+\delta a_{T} r\left(\left\|v_{2}-v_{1}\right\|_{\infty}+\left\|u_{2}-u_{1}\right\|_{\infty}\right),
\end{aligned}
$$

which yields

$$
\begin{aligned}
\| F_{1}\left(u_{2}, v_{2}\right) & -F_{1}\left(u_{1}, v_{1}\right)\left\|_{\infty} \leq a_{T}(\delta r+\sigma)\right\| u_{1}-u_{2} \|_{\infty} \\
& +\delta a_{T} r\left\|v_{1}-v_{2}\right\|_{\infty} .
\end{aligned}
$$

Similarly, one has

$$
\begin{aligned}
& \left|F_{2}\left(u_{2}, v_{2}\right)(t)-F_{2}\left(u_{1}, v_{1}\right)(t)\right| \\
& \leq \frac{\delta}{\Gamma(\beta+v)} \int_{0}^{t}(\psi(t)-\psi(s))^{\beta+v-1} \psi^{\prime}(s) \\
& \quad \times\left(\left|u_{2}(s)\right|\left|v_{2}(s)-v_{1}(s)\right|+\left|v_{1}(s)\right|\left|u_{2}(s)-u_{1}(s)\right|\right) d s \\
& \quad+\frac{\tau}{\Gamma(\beta+v)} \int_{0}^{t}(\psi(t)-\psi(s))^{\beta+v-1} \psi^{\prime}(s)\left|v_{1}(s)-v_{2}(s)\right| d s \\
& \leq \delta b_{T} r\left(\left\|v_{2}-v_{1}\right\|_{\infty}+\left\|u_{2}-u_{1}\right\|_{\infty}\right)+\tau b_{T}\left\|v_{1}-v_{2}\right\|_{\infty},
\end{aligned}
$$

which yields

$$
\begin{aligned}
& \left\|F_{2}\left(u_{2}, v_{2}\right)-F_{2}\left(u_{1}, v_{1}\right)\right\|_{\infty} \\
& \quad \leq \delta b_{T} r\left\|u_{1}-u_{2}\right\|_{\infty}+b_{T}(\delta r+\tau)\left\|v_{1}-v_{2}\right\|_{\infty} .
\end{aligned}
$$

Hence, it follows from (75) and (77) that for all $\left(u_{1}, v_{1}\right)$ ,$\left(u_{2}, v_{2}\right) \in V_{r}$,

$$
d\left(F\left(u_{1}, v_{1}\right), F\left(u_{2}, v_{2}\right)\right)_{2} \cdot M d\left(\left(u_{1}, v_{1}\right),\left(u_{2}, v_{2}\right)\right),
$$

where

$$
\mathscr{M}=\left(\begin{array}{cc}
a_{T}(\delta r+\sigma) & \delta a_{T} r \\
\delta b_{T} r & b_{T}(\delta r+\tau)
\end{array}\right) .
$$

On the other hand, the characteristic polynomial of $\mathscr{M}$ is given by

$$
Q(\lambda)=\left[a_{T}(\delta r+\sigma)-\lambda\right]\left[b_{T}(\delta r+\tau)-\lambda\right]-\delta^{2} a_{T} b_{T} r^{2} .
$$

An elementary calculation shows that the polynomial function $Q$ admits two roots (which are the eigenvalues of $\mathscr{M})$ given by

$$
\begin{aligned}
\lambda_{1}(r)= & \frac{a_{T}(\sigma+\delta r)+b_{T}(\tau+\delta r)}{2} \\
& -\frac{1}{2}\left[\left(\left(a_{T} \sigma-b_{T} \tau\right)+\left(a_{T}-b_{T}\right) \delta r\right)^{2}+4 a_{T} b_{T} \delta^{2} r^{2}\right]^{\frac{1}{2}}, \\
\lambda_{2}(r)= & \frac{f(r)}{2} .
\end{aligned}
$$

It can be easily seen that

$$
0 \leq \lambda_{1}(r) \leq \lambda_{2}(r)
$$

which yields

$$
\rho(\mathscr{M})=\frac{f(r)}{2} .
$$

Since $f(r)<2$ (by (vii)), it holds that $\rho(\mathscr{M})<1$. Therefore, by Lemma 11, the mapping $F$ admits a fixed point $\left(u^{*}\right.$ , $\left.v^{*}\right) \in V_{r} \subset V$, which is a solution to the system of integral equation (32). Moreover, for all $\left(f_{0}, g_{0}\right) \in V_{r}$, the Picard sequence $\left\{\left(f_{n}, g_{n}\right)\right\}$ defined by $\left(f_{n+1}, g_{n+1}\right)=F\left(f_{n}, g_{n}\right)=($ $\left.F_{1}\left(f_{n}, g_{n}\right), F_{2}\left(f_{n}, g_{n}\right)\right)$ converges uniformly to $\left(u^{*}, v^{*}\right)$. On the other hand, by Proposition 14, we know that (32) admits at least one solution in $V$. Hence, one deduces that $\left(u^{*}, v^{*}\right)$ is the only solution to (32) in $V$.

\section{Some Special Cases}

Some special cases of Theorem 15 are discussed in this section. We first consider the case $\alpha+\mu=\beta+v:=\xi$. In this case, 
one has

$$
a_{T}=b_{T}=\frac{(\psi(T)-\psi(0))^{\xi}}{\Gamma(\xi+1)}:=c_{T} .
$$

Corollary 16. Suppose that the following conditions are satisfied:

$$
\begin{gathered}
\left(A_{1}\right) T>0, \psi \in \Psi \\
\left(A_{2}\right) 0<\alpha, \beta<1, \alpha+\mu=\beta+v:=\xi \geq 1 \\
\left(A_{3}\right) \kappa, \sigma, \delta, \tau>0 \\
\left(A_{4}\right) 4 \tau \leq \sqrt{\sigma^{2}+4 \delta \kappa}-\sigma \\
\left(A_{5}\right) u_{0}=\frac{\tau}{\delta}, 0 \leq v_{0} \leq u_{0} \\
\left(A_{6}\right) C_{T}<\min \left\{\frac{\tau}{\delta \kappa}, \frac{1}{\sqrt{\sigma^{2}+4 \delta \kappa}-\sigma}, \frac{2}{\sigma+5 \tau+\sqrt{(\sigma-\tau)^{2}+16 \tau^{2}}}\right\}
\end{gathered}
$$

Then, the system of integral equation (32) admits one and only one solution $\left(u^{*}, v^{*}\right) \in V$. Moreover, for all $f_{0}, g_{0}$ $\in V$ satisfying $u_{0} \leq f_{0},\left\|f_{0}\right\|_{\infty} \leq 2 \tau / \delta$ and $\left\|g_{0}\right\|_{\infty} \leq 2 \tau / \delta$, the sequence $\left\{\left(f_{n}, g_{n}\right)\right\} \subset V$ defined by (52) converges uniformly to $\left(u^{*}, v^{*}\right)$.

Proof. The results follow immediately by taking $r=2 \tau / \delta$ and $\alpha+\mu=\beta+\nu$ in Theorem 15.

Consider now the special case of Corollary 16 when $\psi(t$ )$=t$.

Corollary 17. Suppose that the following conditions are satisfied:

$$
\begin{gathered}
\left(B_{1}\right) 0<\alpha, \beta<1, \alpha+\mu=\beta+\nu:=\xi \geq 1 \\
\left(B_{2}\right) \kappa, \sigma, \delta, \tau>0 \\
\left(B_{3}\right) 4 \tau \leq \sqrt{\sigma^{2}+4 \delta \kappa}-\sigma \\
\left(B_{4}\right) u_{0}=\frac{\tau}{\delta}, 0 \leq v_{0} \leq u_{0} \\
\left(B_{4}\right) 0<T<\left(\Gamma(\xi+1) \min \left\{\frac{\tau}{\delta \kappa}, \frac{1}{\sqrt{\sigma^{2}+4 \delta \kappa}-\sigma}, \frac{2}{\sigma+5 \tau+\sqrt{(\sigma-\tau)^{2}+16 \tau^{2}}}\right\}\right)^{1 / \xi}
\end{gathered}
$$

Then, the system of integral equation (32) admits one and only one solution $\left(u^{*}, v^{*}\right) \in V$. Moreover, for all $f_{0}, g_{0} \in V$ satisfying $u_{0} \leq f_{0},\left\|f_{0}\right\|_{\infty} \leq 2 \tau / \delta$ and $\left\|g_{0}\right\|_{\infty} \leq 2 \tau / \delta$, the sequence $\left\{\left(f_{n}, g_{n}\right)\right\} \subset V$ defined by (52) converges uniformly to $\left(u^{*}\right.$, $\left.v^{*}\right)$.
Proof. In this case, one has

$$
C_{T}=\frac{T^{\xi}}{\Gamma(\xi+1)} .
$$

Therefore, the assumption $\left(A_{6}\right)$ in Corollary 16 reduces to the assumption $\left(B_{5}\right)$. Hence, by Corollary 16 , the desired results follow.

We take now $\psi(t)=t$ and consider the limit cases $(\alpha, \mu)$ $\rightarrow\left(1^{-}, 0^{+}\right)$and $(\beta, v) \rightarrow\left(1^{-}, 0^{+}\right)$. Notice that in this case, the system of integral equation (32) reduces to

$$
\left\{\begin{array}{l}
u(t)=u_{0}+\int_{0}^{t}(\kappa-\sigma u(s)-\delta u(s) v(s)) d s, \quad 0 \leq t \leq T \\
v(t)=v_{0}+\int_{0}^{t}(\delta u(s) v(s)-\tau v(s)) d s, \quad 0 \leq t \leq T,
\end{array}\right.
$$

which corresponds to the integral representation of the system of ordinary differential equations (1) subject to the initial conditions (3).

Taking $\xi=1$ in Corollary 17, one deduces the following results.

Corollary 18. Suppose that the following conditions are satisfied:

$$
\left(C_{1}\right) \kappa, \sigma, \delta, \tau>0
$$

$$
\begin{aligned}
& \left(C_{2}\right) 4 \tau \leq \sqrt{\sigma^{2}+4 \delta \kappa}-\sigma \\
& \left(C_{3}\right) u_{0}=\frac{\tau}{\delta}, 0 \leq v_{0} \leq u_{0}
\end{aligned}
$$

$\left(C_{4}\right) 0<T<\min \left\{\frac{\tau}{\delta \kappa}, \frac{1}{\sqrt{\sigma^{2}+4 \delta \kappa}-\sigma}, \frac{2}{\sigma+5 \tau+\sqrt{(\sigma-\tau)^{2}+16 \tau^{2}}}\right\}$

Then, the system of integral equation (88) admits one and only one solution $\left(u^{*}, v^{*}\right) \in V$. Moreover, for all $f_{0}, g_{0} \in V$ satisfying $u_{0} \leq f_{0},\left\|f_{0}\right\|_{\infty} \leq 2 \tau / \delta$ and $\left\|g_{0}\right\|_{\infty} \leq 2 \tau / \delta$, the sequence $\left\{\left(f_{n}, g_{n}\right)\right\} \subset V$ defined by

$$
\left\{\begin{array}{l}
f_{n+1}(t)=\mu_{0}+\int_{0}^{t}\left(\kappa-\sigma f_{n}(s)-\delta f_{n}(s) g_{n}(s)\right) d s, \quad 0 \leq t \leq T, \\
g_{n+1}(t)=v_{0}+\int_{0}^{t}\left(\delta f_{n}(s) g_{n}(s)-\tau g_{n}(s)\right) d s, \quad 0 \leq t \leq T,
\end{array}\right.
$$

converges uniformly to $\left(u^{*}, v^{*}\right)$.

\section{Data Availability}

No data were used to support this study. 


\section{Conflicts of Interest}

The authors declare that they have no conflicts of interest.

\section{Authors' Contributions}

All authors contributed equally and significantly in writing this article. All authors read and approved the final manuscript.

\section{Acknowledgments}

The authors extend their appreciation to the Deputyship for Research \& Innovation, Ministry of Education-Kingdom of Saudi Arabia for funding this research work through the project number IFKSURG-237.

\section{References}

[1] K. L. Packer, HIV infection: the facts you need to know, Franklin Watts, 1998.

[2] R. A. Weiss, "How does HIV cause AIDS?," Science, vol. 260, no. 5112, pp. 1273-1279, 1993.

[3] S. Bonhoeffer, J. M. Coffin, and M. A. Nowak, "Human immunodeficiency virus drug therapy and virus load," Journal of Virology, vol. 71, no. 4, pp. 3275-3278, 1997.

[4] H. C. Tuckwell and F. Y. M. Wan, "On the behavior of solutions in viral dynamical models," Biosystems, vol. 73, no. 3, pp. 157-161, 2004.

[5] A. V. M. Herz, S. Bonhoeffer, R. M. Anderson, R. M. May, and M. A. Nowak, "Viral dynamics in vivo: limitations on estimates of intracellular delay and virus decay," Proceedings of the National Academy of Sciences of the United States of America, vol. 93, no. 14, pp. 7247-7251, 1996.

[6] A. Perelson, A. Neumann, M. Markowitz, J. Leonard, and D. Ho, "HIV-1 dynamics in vivo: virion clearance rate, infected cell life-span, and viral generation time," Science, vol. 271, no. 5255, pp. 1582-1586, 1996.

[7] L. Rong, M. A. Gilchrist, Z. Feng, and A. S. Perelson, "Modeling within-host HIV-1 dynamics and the evolution of drug resistance: trade-offs between viral enzyme function and drug susceptibility," Journal of Theoretical Biology, vol. 247, no. 4, pp. 804-818, 2007.

[8] A. R. McLean, V. C. Emery, A. Webster, and P. D. Griffiths, "Population dynamics of HIV within an individual after treatment with zidovudine," AIDS, vol. 5, no. 5, pp. 485-490, 1991.

[9] L. Debnath, "Recent applications of fractional calculus to science and engineering," International Journal of Mathematics and Mathematical Sciences, vol. 54, 3442 pages, 2003.

[10] A. Kilbas, H. Srivastava, and J. Trujillo, Theory and Applications of Fractional Differential Equations, Elsevier, Amsterdam, 2006.

[11] C. P. Li, Y. Q. Chen, and J. Kurths, "Fractional calculus and its applications," Philosophical Transactions of the Royal Society of London A: Mathematical, Physical and Engineering Sciences, vol. 371, article 20130037, no. 1990, p. 3, 2013.

[12] Z. H. Wang, "Fractional calculus: a mathematical method in describing the memory and process of materials," Scientific Chinese, vol. 3, pp. 76-78, 2011.
[13] A. A. M. Arafa, S. Z. Rida, and M. Khalil, "Fractional modeling dynamics of HIV and CD4 $4^{+}$T-cells during primary infection," Nonlinear Biomedical Physics, vol. 6, no. 1, pp. 1-7, 2012.

[14] Y. Ding and H. Ye, "A fractional-order differential equation model of HIV infection of CD4+ T-cells," Mathematical and Computer Modelling, vol. 50, no. 3-4, pp. 386-392, 2009.

[15] C. M. A. Pinto and A. R. M. Carvalho, "A latency fractional order model for HIV dynamics," Journal of Computational and Applied Mathematics, vol. 312, pp. 240-256, 2017.

[16] Y. Yan and C. Kou, "Stability analysis for a fractional differential model of HIV infection of CD4+T-cells with time delay," Mathematics and Computers in Simulation, vol. 82, no. 9, pp. 1572-1585, 2012.

[17] Y. Wang, L. Liu, X. Zhang, and Y. Wu, "Positive solutions of an abstract fractional semipositone differential system model for bioprocesses of HIV infection," Applied Mathematics and Computation, vol. 258, pp. 312-324, 2015.

[18] R. Almeida, "A Caputo fractional derivative of a function with respect to another function," Communications in Nonlinear Science and Numerical Simulation, vol. 44, pp. 460-481, 2017.

[19] A. D. Filip and A. Petruşel, "Fixed point theorems on spaces endowed with vector-valued metrics," Fixed Point Theory and Applications, vol. 2010, Article ID 281381, 16 pages, 2010.

[20] L. C. Piccinini, G. Stampacchia, and G. Vidossich, Ordinary Differential Equations in $\mathbb{R}^{n}$, Problems and Methods, Springer-Verlag, NY, 1984. 